\title{
An Efficient Technique based on Polynomial Chaos to Model the Uncertainty in the Resonance Frequency of Textile Antennas due to Bending
}

\author{
Freek Boeykens, Hendrik Rogier, Senior Member IEEE, and Luigi Vallozzi
}

\begin{abstract}
The generalized polynomial chaos theory is combined with a dedicated cavity model for curved textile antennas to statistically quantify variations in the antenna's resonance frequency under randomly varying bending conditions. The nonintrusive stochastic method solves the dispersion relation for the resonance frequencies of a set of radius of curvature realizations corresponding to the Gauss quadrature points belonging to the orthogonal polynomials having the probability density function of the random variable as a weighting function. The formalism is applied to different distributions for the radius of curvature, either using a priori known or on the fly constructed sets of orthogonal polynomials. Numerical and experimental validation shows that the new approach is at least as accurate as Monte Carlo simulations while being at least 100 times faster. This makes the method especially suited as design tool to account for performance variability when textile antennas are deployed on persons with varying body morphology.
\end{abstract}

Index Terms-Microstrip antennas, Random variables, Statistical analysis, flexible electronics, textile antennas

\section{INTRODUCTION}

In the last decade, we witnessed a rapid growth of smart textiles and interactive fabrics, mainly through the integration of electronic circuits capable of sensing and communicating with their environment. As key components of these systems, the design of textile antennas deserves special care [1]-[4]. Wearable antenna garments are subjected to bending and crumpling [5]. Moreover, their radiation efficiency might drop due to proximity of the human body. Whereas the latter is easily countered by adopting a microstrip patch antenna topology with a sufficiently large ground plane, the former is typically mitigated by overspecifying the requirements for the planar antenna. The designer then aims for a larger bandwidth and a higher return loss than actually required in the intended application. Yet, this approach may increase effects of out-ofband interference as well as cost. A more judicious design requires that deterministic full-wave electromagnetic solvers be extended with stochastic methods that statistically quantify randomness in the antenna's figures of merit. These design tools then capture the effect of uncertainty in the wearable antenna's performance parameters due to variations in, for example, the radius of curvature of the flexible antenna caused by

F. Boeykens, H. Rogier and L. Vallozzi are with the Department of Information Technology, Ghent University, St. Pietersnieuwstraat 41, 9000 Ghent, Belgium. E-mail: freek.boeykens@intec.ugent.be, hendrik.rogier@intec.ugent.be, luigi.vallozzi@intec.ugent.be. H. Rogier is also a guest professor at the INTEC Associated Lab of IMEC, Belgium. E-mail: hendrik.rogier@imec.be.
1) movements and activity of the person wearing the antenna;

2) variations in body morphology over many different users.

This paper specifically focuses on the second scenario, as our experiments demonstrated that movements in the first scenario do not lead to large variations in the curvature radius, resulting in a negligible shift of the resonance frequency.

As Monte-Carlo simulations require many realizations to accurately describe the statistics of the random process, they are too time-consuming to be applied as design tools. Therefore, the generalized polynomial chaos formalism [6], [7] was introduced as a more effective approach to rapidly model uncertainty due to variations in the input parameters. Recently, in addition to techniques that quickly determine statistical moments [8], [9], many new intrusive and nonintrusive procedures were proposed [10] and applied to transmission lines [11]-[15], multiport microwave circuits [16], [17], scattering [18]-[20] and antennas [15]. Yet, none of them is dedicated to wearable antenna design. In particular, the proposed techniques effectively account for simple variations in geometry, but become very complex when modeling curved antennas. Hence, they are unsuited to characterize the uncertainty on the resonance frequency due to randomness in the radius of curvature. For this problem, the stochastic full-wave approach is still too CPU-intensive and a designer needs to rely on a faster, albeit more approximate, technique to estimate the probability density function of the antenna performance due to geometrical variations.

Therefore, we propose a new efficient statistical design formalism that combines a dedicated cavity model for curved textile patch antennas with a non-intrusive generalized polynomial chaos algorithm to calculate the statistical distribution of the resonance frequency. In Section II the non-intrusive polynomial chaos theory is applied to derive the statistical properties of the resonance frequencies of the textile patch antenna for a given statistical distribution of the radius of curvature. Section III first provides a numerical verification for a Gaussian, uniform and truncated Gaussian distribution of the radius of curvature, by comparing the probability density function obtained with the new method to the distribution found by applying Monte Carlo simulations. Moreover, the technique is experimentally validated by the real-life example of a textile patch antenna on a human arm. The conclusions are summarized in Section IV. 


\section{STATISTICS OF THE RESONANCE FREQUENCY OF A CURVED TEXTILE PATCH ANTENNA}

The new formalism outlined in this section provides a fast and accurate description of the statistics of the resonance frequency thanks to

1) the use of a recently proposed cavity model for curved microstrip patch antennas [21] that empirically accounts for textile substrate compression and subsequent change in substrate height and permittivity;

2) the application of a non-intrusive generalized polynomial chaos formalism [22] using the solutions of the dispersion equation of the cavity model for a predefined set of radii of curvature. This set consists of the quadrature points corresponding to the orthogonal polynomials having as a weighting function the probability density function of the input random variable.

These orthogonal polynomials are either readily available, such as the Legendre polynomials for a uniform distribution, or, for an arbitrary distribution [23], constructed on the fly by applying the Modified Chebyshev algorithm [24] followed by the Golub-Welsch algorithm [25] to obtain the quadrature points and weights.

\section{A. Non-intrusive generalized polynomial chaos}

Assume that an input random variable $X$, in our case the radius of curvature of a bent textile antenna, is given and that we want to determine the corresponding variation of the output variable $Y$, in our case the resonance frequency of the patch. The statistical variation of $X$ is described by the cumulative distribution function $\mathcal{P}^{X}$ and probability density function $d \mathcal{P}^{X}$ in the sample space $\Omega$. To determine the statistics of $Y$, we rely on the Wiener-Askey scheme [10] to approximate the transformation $Y=f(X)$ by the following polynomial expansion of order $P$

$$
Y \approx f^{P}(X)=\sum_{k=0}^{P} y_{k}^{X} \phi_{k}^{X}(X) .
$$

An optimal expansion is obtained when the set of expansion polynomials forms a complete orthogonal basis in $\Omega$ with orthogonality relation

$$
\begin{aligned}
\left\langle\phi_{i}^{X}(x), \phi_{j}^{X}(x)\right\rangle & =\int_{\Omega} \phi_{i}^{X}(x) \phi_{j}^{X}(x) d \mathcal{P}^{X}(x) \\
& =\left\langle\left(\phi_{i}^{X}\right)^{2}\right\rangle \delta_{i j} .
\end{aligned}
$$

In this case, the Cameron-Martin convergence theorem ensures exponential convergence to the function $Y=f(X)$ for $P \rightarrow$ $\infty$. By relying on the Askey scheme, we can directly make use of several families of orthogonal polynomials corresponding to well-established distributions $d \mathcal{P}^{X}(x)$, such as, for example, Hermite polynomials for the Gaussian distribution and Legendre polynomials for the uniform distribution. To determine the unknown expansion coefficients $y_{k}^{X}$, we apply Galerkin weighting to (1) and make use of (2) to obtain

$$
y_{j}^{X}=E\left[Y(x) \phi_{j}^{X}(x)\right]=\int_{\Omega} Y(x) \phi_{j}^{X}(x) d \mathcal{P}^{X}(x) .
$$

We can now conveniently approximate the integral by a suitable $N$-point Gauss quadrature rule, being

$$
y_{j}^{X} \approx \sum_{i=1}^{N} w_{i} Y\left(x_{i}\right) \phi_{j}^{X}\left(x_{i}\right), \quad j=0,1, \ldots, P ;
$$

where the quadrature points $x_{i}$ are given by the $N$ zeros of $\phi_{N}^{X}(x)$ in $\Omega$ and with $w_{i}$ the corresponding weights. In order to evaluate (4), we must calculate $Y=f(X)$ for $N$ realizations of the random variable $X$ corresponding to the quadrature points. In contrast to intrusive methods, this technique may be applied to any function or procedure taking $x_{i}$ as an input and yielding $Y\left(x_{i}\right)$ as an output, however complex this formalism may be. In this paper, we apply the non-intrusive formalism to the cavity model for textile antennas subjected to cylindrical bending, proposed in [21] and summarized in the next section.

Note that, in order to evaluate the moments of the output distribution $\mathcal{P}^{Y}$, we do not need to revert to (1), as they might be directly computed based on the knowledge of the $Y\left(x_{i}\right)$ generated in order to calculate (4). Indeed, we have that

$$
\begin{aligned}
E[g(y)] & =\int_{\Omega_{y}} g(y) d \mathcal{P}^{Y}(y) \\
& =\int_{\Omega} g(Y(x)) d \mathcal{P}^{X}(x) \approx \sum_{i=1}^{N} w_{i} g\left[Y\left(x_{i}\right)\right],
\end{aligned}
$$

for an arbitrary function $g(y)$.

\section{B. Cavity model for textile antennas subjected to cylindrical bending}

Consider a rectangular patch of length $L$, width $W$, deployed on a textile substrate of thickness $h$ (measured in planar state following the ISO 5084 standard) and permittivity $\epsilon_{r}$, subjected to bending around a cylinder with angle of curvature $R$, as shown in Fig. 1. We first consider bending in the direction of the patch width $W_{p}$ (defined in Fig. 2). In Fig. 1 we then set $L=L_{p}$ and $W=W_{p}$. The resonance frequency satisfies the transcendental equation

$$
J_{k_{\phi}}^{\prime}\left(k_{\rho} R\right) Y_{k_{\phi}}^{\prime}\left(k_{\rho}(R+h)\right)=J_{k_{\phi}}^{\prime}\left(k_{\rho}(R+h)\right) Y_{k_{\phi}}^{\prime}\left(k_{\rho} R\right),
$$

with $k_{\phi}=\frac{m \pi}{\beta}, k_{z}=\frac{n \pi}{L}, k_{\rho}=\sqrt{k^{2}-k_{z}^{2}}$. The angle $\beta$ follows from the relationship $W=\beta(R+h d)$ with $d=0.5$ for perfectly stretchable patches and $d=1$ for completely non-stretchable patches. In addition, specifically for textile substrates, compression of the antenna substrate may occur, resulting in a variation of the permittivity compared to planar state. This is accounted for by setting $\epsilon_{r, \text { comp }}=\epsilon_{r, \text { flat }}\left(1+\eta \frac{h[m m](d-0.5)}{R[m]}\right)$, where $\eta$ is a substratedependent empirical parameter, determined by measurements on actual textile antenna prototypes subjected to bending. Hence, for non-magnetic substrates, resonance frequency $f_{r}$ of the bent patch and wave number $k$ are related by $k=$ $2 \pi f_{r} \sqrt{\mu_{0} \epsilon_{0} \epsilon_{r, \mathrm{comp}}}$.

For bending along $L_{p}$, as defined in Fig. 2, we set $L=W_{p}$ and $W=L_{p}$ in Fig. 1. Instead of (6), the resonance frequency now satisfies

$$
J_{k_{\phi}}\left(k_{\rho} R\right) Y_{k_{\phi}}\left(k_{\rho}(R+h)\right)=J_{k_{\phi}}\left(k_{\rho}(R+h)\right) Y_{k_{\phi}}\left(k_{\rho} R\right) .
$$




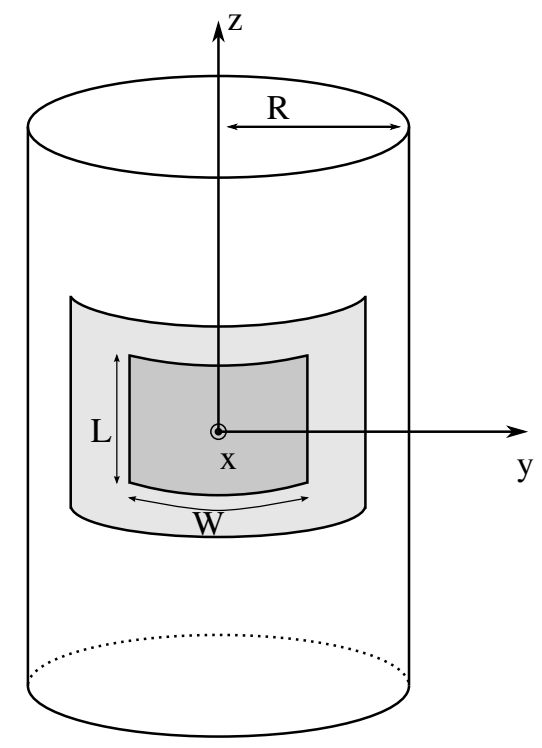

(a)

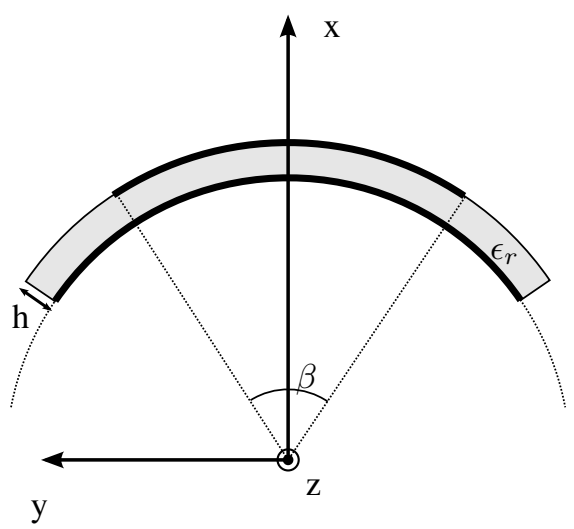

(b)

Fig. 1. Geometry of the cavity: top view (a) and side view (b).

C. On-the-fly construction of the orthogonal polynomials $\phi_{j}^{X}(x)$

Although the Askey scheme provides analytic expressions for sets of orthogonal polynomials corresponding to many widely-used distributions, many practical cases exist where the required set is not readily available for the distribution at hand. Assume, for example, that the radius of curvature is distributed following a normal distribution. The infinite support of the Gauss distribution is not compatible with the practical fact that textile antennas deployed on the human body only exhibit a positive radius of curvature. This means that, in practice, we must restrict the normal distribution to a finite support. In this case, we do not have a set of orthogonal polynomials readily available. Therefore, we on the fly construct the set of polynomials that are orthogonal to the truncated gaussian distribution as a weighting function. Although many techniques exist to orthogonalize monic polynomials, the problem is known to be notoriously ill-conditioned. We start from the set of Hermite polynomials corresponding to the Gauss distribution with infinite support and use the Modified Chebyshev algorithm [24] to transform the recurrence relation for Hermite polynomials

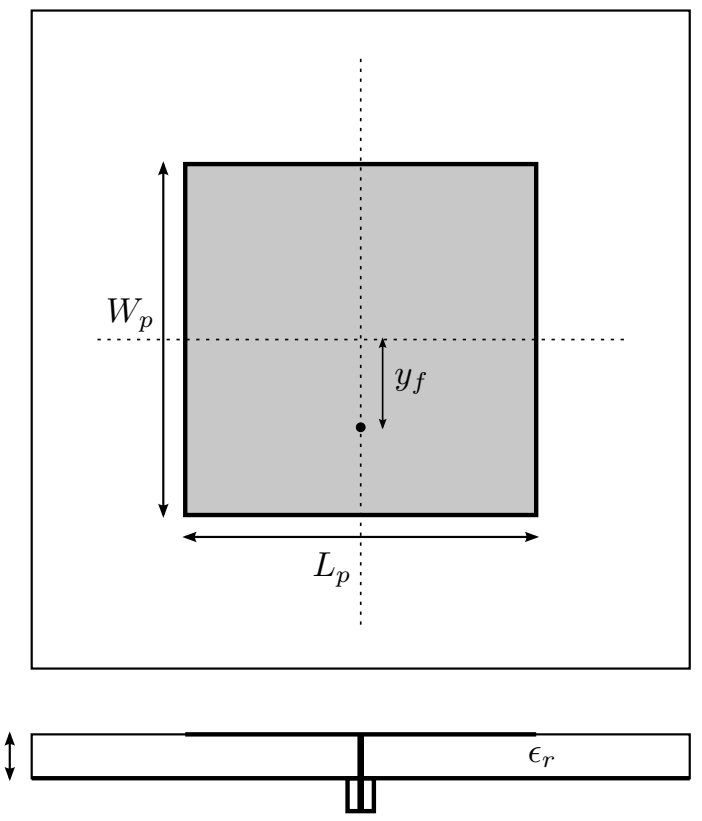

Fig. 2. Geometry of a prototype textile antenna.

to a recurrence relationship for polynomials orthogonal with respect to the truncated normal distribution. Next, we apply the Golub-Welsch algorithm [25] to derive the quadrature points $x_{i}$ and weights $w_{i}$ required to evaluate (4). This procedure can be used to construct a set of orthogonal polynomials for any arbitrary distribution of the input random variables.

\section{RESUlTS}

We now illustrate our approach for different textile patch antenna prototypes subjected to cylindrical bending when deployed on an arm or a leg. The ability to isolate variations in the antenna's figures of merit solely due to bending is an important asset of the non-intrusive polynomial chaos approach. Indeed, conventional techniques that merely rely on measurements of textile antenna prototypes can only characterize the overall variations due to the aggregate effect of many different adverse factors. To keep the paper concise, we concentrate on bending in the direction of the patch width $W_{p}$, solving transcendental equation (6), since bending in this direction gives rise to the largest variation in the resonance frequency of the antenna. However, if one is also interested in studying or adding effects originating from fabrication tolerances, humidity, temperature, etc., it is sufficient to replace the dispersion relation by a different relation that takes these effects into account. This new relation is then directly integrated into the stochastic framework. In a similar fashion, variations in the figures of merit of other antenna types, such as dipole antennas, may be studied.

First, our technique based on generalized polynomial chaos is numerically validated in terms of speed and accuracy by comparison to a Monte Carlo approach for different distributions of the radius of curvature. Second, experimental validation is performed by considering a real-life example. It is also shown that the distributions and their parameters used 
TABLE I

DESIGN PARAMETERS OF THE PROTOTYPES.

\begin{tabular}{|c||c|c|c|c|c|}
\hline Prototype & 1 & 2 & 3 & 4 & 5 \\
\hline \hline $\mathrm{L}_{\mathrm{p}}[\mathrm{mm}]$ & 81.2 & 81.2 & 78.5 & 52.5 & 78.5 \\
\hline $\mathrm{W}_{\mathrm{p}}[\mathrm{mm}]$ & 69.25 & 69.25 & 69.3 & 43.7 & 68.3 \\
\hline $\mathrm{h}[\mathrm{mm}]$ & 2 & 2 & 2.7 & 2.7 & 4.05 \\
\hline $\mathrm{y}_{\mathrm{f}}[\mathrm{mm}]$ & 16 & 16 & 17 & 11 & 16.8 \\
\hline$\epsilon_{r}$ & 1.75 & 1.75 & 1.715 & 1.715 & 1.715 \\
\hline $\mathrm{d}$ & 0.5 & 1 & 1 & 1 & 1 \\
\hline
\end{tabular}

during the numerical validation indeed correspond to real-life applications.

\section{A. Numerical validation}

Let us consider the five prototypes studied in [21], constructed on different flexible materials and operating at different frequencies. All prototypes are probe-fed microstrip patch antennas, with the probe located on the perpendicular bisector of the $L_{p}$ edge, as shown in Fig. 2. The dimensions of each prototype are listed in Table I. Prototypes 1 and 2 are implemented on an aramid fabric as antenna substrate. The patch material of prototype 1 is the stretchable electro-textile Flectron ${ }^{\circledR}$, whereas non-stretchable copper foil is applied for prototype 2. Prototypes 3, 4 and 5 are fabricated on a cotton substrate using copper foil as patch material. Prototypes 1, 2, 3 and 5 exhibit a sharp resonance peak close to the $1.57 \mathrm{GHz}$ GPS band, whereas prototype 4 resonates in the vicinity of the $2.45 \mathrm{GHz}$ ISM band. Prototypes 3 and 5 have a different substrate thickness. The parameters of the prototypes are summarized in Table I, where the substrate height $h$ is determined based on the ISO 5084 standard [26]. The resonance frequencies of all prototypes were measured for different bending radii to construct the dispersion relation following Section II-B.

We consider two nominal application scenarios where the prototype 2 textile antenna is bent in the direction of the patch width $W_{p}$ : a textile antenna deployed on the human leg, with an average radius of curvature $\bar{R}=10 \mathrm{~cm}$ and a flexible antenna deployed on the human arm, corresponding to a mean radius of curvature $\bar{R}=5 \mathrm{~cm}$. We now assume that, due to variations in body morphology, the radius of curvature behaves as a random variable following a Gaussian distribution. Due to the infinite support of the normal distribution, we need to restrict the range of acceptable values for the standard deviation in order to avoid unrealistic (negative) values for the radius of curvature. Larger variations in the radius of curvature can be considered by assuming a uniform distribution, by relying on a set of Legendre polynomials, or a truncated Gaussian distribution, using a set of on-the-fly constructed orthogonal polynomials following Section II-C. Let us first compare the distributions obtained for prototype 2 based on non-intrusive generalized polynomial chaos and Monte Carlo simulations. Fig. 3 presents the cumulative distribution functions (CDFs) obtained for the textile antenna deployed on a human arm with a mean radius of curvature $\bar{R}=5 \mathrm{~cm}$, considering a Gaussian distribution with standard deviation $\sigma=7 \mathrm{~mm}$, a



Fig. 3. CDFs of the resonance frequency for the prototype 2 textile antenna deployed on the arm with a mean radius of curvature $\bar{R}=5 \mathrm{~cm}$, assuming a Gaussian distribution with standard deviation $\sigma=7 \mathrm{~mm}$, a uniform distribution over the interval $[1 \mathrm{~cm}, 9 \mathrm{~cm}]$, and a Gaussian distribution truncated to the interval $[2 \mathrm{~cm}, 9 \mathrm{~cm}]$ with standard deviation $\sigma=2 \mathrm{~cm}$.

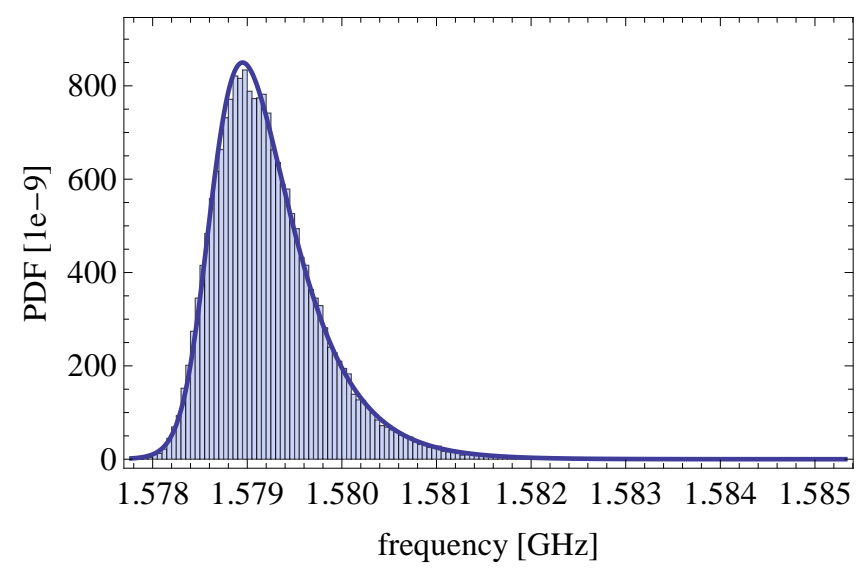

Fig. 4. PDF of the resonance frequency for the prototype 2 textile antenna deployed on the leg assuming a Gaussian distributed radius of curvature with mean $\bar{R}=10 \mathrm{~cm}$ and standard deviation $\sigma=17 \mathrm{~mm}$.

uniform distribution over the interval $[1 \mathrm{~cm}, 9 \mathrm{~cm}]$, and a Gaussian distribution restricted to the interval $[2 \mathrm{~cm}, 9 \mathrm{~cm}]$ with standard deviation $\sigma=2 \mathrm{~cm}$. An excellent agreement is found between our non-intrusive generalized polynomial chaos method and the Monte Carlo approach. Yet, on the one hand, to apply the Monte Carlo technique, the dispersion relation (6) is evaluated for 50000 realizations on an Intel(R) Core(TM) i72760QM CPU operating at $2.40 \mathrm{GHz}$ with 8 GB RAM using Mathematica 8.0, taking $4 \min 51 \mathrm{~s}, 5 \min 21 \mathrm{~s}$, and $5 \min 10 \mathrm{~s}$ of CPU-time for the Gaussian, uniform and truncated Gaussian distributions, respectively. In order to apply the non-intrusive polynomial chaos, on the other hand, adopting a conservative strategy for all distributions considered, we evaluated (4) at 33 quadrature points, requiring $1.4 \mathrm{~s}$ to compute the dispersion relation (6) at these points.

Fig. 4 presents the probability density function (PDF) when the prototype 2 textile antenna is deployed on a human leg and subjected to bending following a Gaussian distributed radius of curvature along the patch width $W_{p}$ with mean $\bar{R}=10$ $\mathrm{cm}$ and standard deviation $\sigma=17 \mathrm{~mm}$. For this small $\sigma$, 


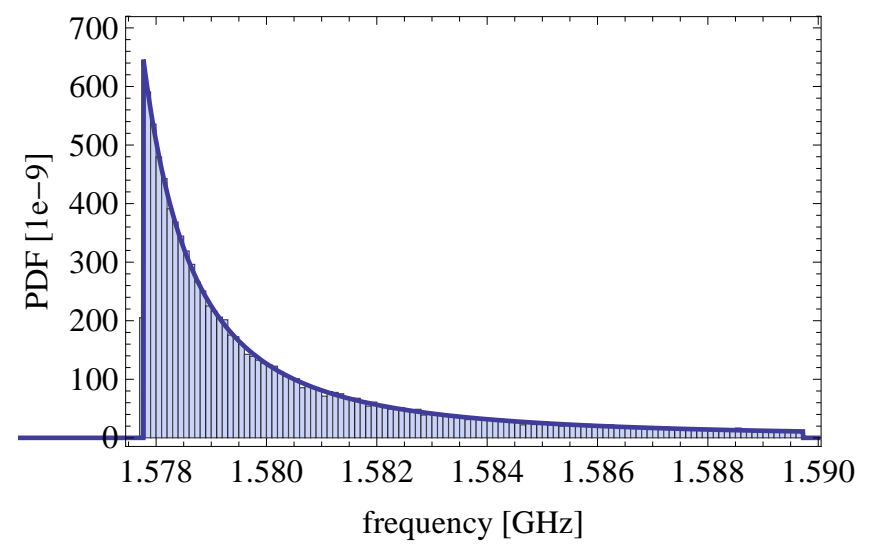

Fig. 5. PDF of the resonance frequency for the prototype 2 textile antenna deployed on the leg considering a uniformly distributed radius of curvature in the interval $[2 \mathrm{~cm}, 18 \mathrm{~cm}]$.

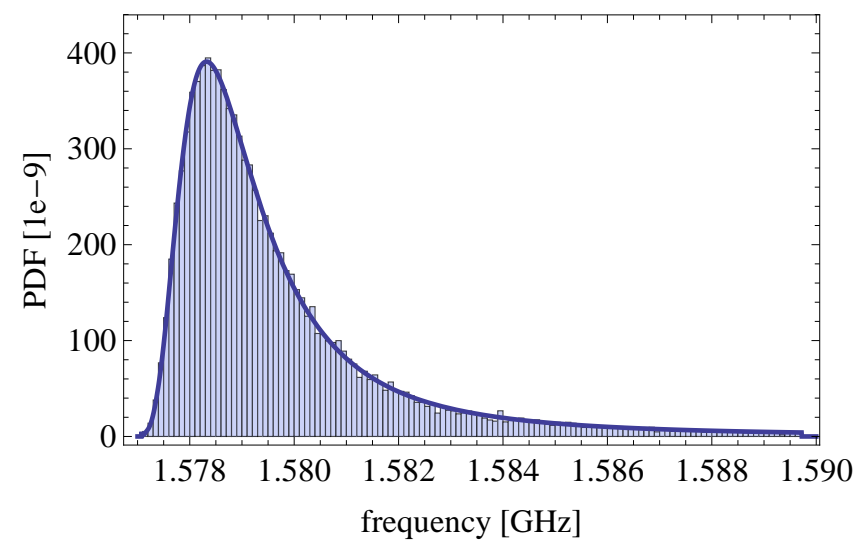

Fig. 6. PDF of the resonance frequency for the prototype 2 textile antenna deployed on the leg considering a Gaussian distributed radius of curvature truncated to the interval $[2 \mathrm{~cm}, 30 \mathrm{~cm}]$ with mean $\bar{R}=10 \mathrm{~cm}$ and standard deviation $\sigma=5 \mathrm{~cm}$.

the distribution of the resonance frequency does not differ that much from a Gaussian distribution. Due to the non-linear dependence of the resonance frequency on the bending radius, we see that the output PDF exhibits a slight positive skew of 1.319 (1.325 based on the Monte Carlo simulation). Yet, we notice a good agreement with the Monte Carlo simulation when using Hermite polynomials up to order $P=3$ in the Wiener-Askey expansion (1). When the radius of curvature is uniformly distributed over an interval of width $16 \mathrm{~cm}$ centered around $\bar{R}=10 \mathrm{~cm}$, we observe in Fig. 5 that the output PDF for the resonance frequency differs a lot from the uniform input distribution. The skewness of the output distribution has now increased to 1.579 (an identical value is found using Monte Carlo simulation). We obtain a good agreement between the Monte-Carlo simulation and the generalized polynomial chaos based on Legendre polynomials of orders up to $P=21$. Next, we consider a Gaussian distribution around the mean bending radius $\bar{R}=10 \mathrm{~cm}$ that is restricted to the interval $[2 \mathrm{~cm}, 30 \mathrm{~cm}]$. As the distribution's support is finite, compared to the complete Gaussian distribution, we may now increase the standard deviation to $\sigma=5 \mathrm{~cm}$. For illustrative purposes, we deliberately chose to make the input

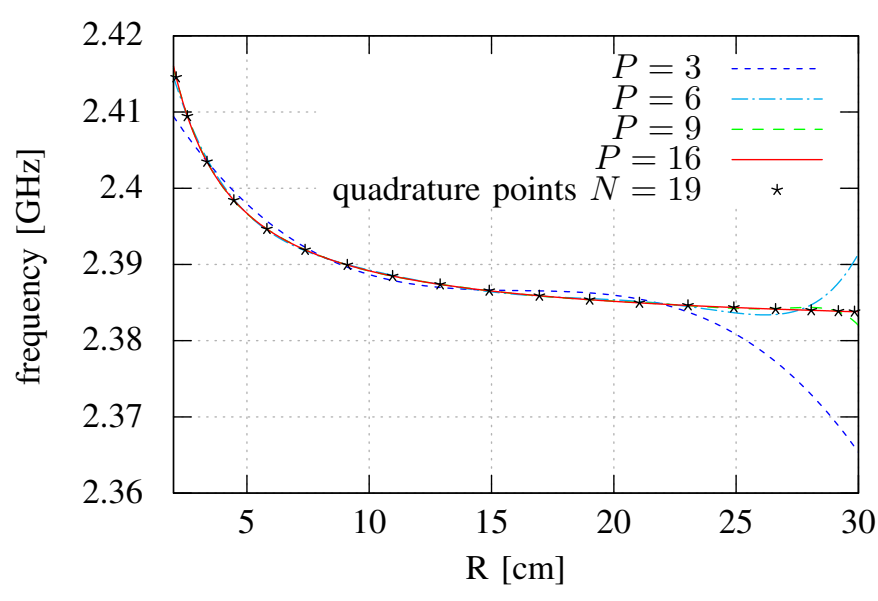

Fig. 7. Prototype 4 bent around a human leg with a radius of curvature following a Gaussian distribution truncated to the interval $[2 \mathrm{~cm}, 30 \mathrm{~cm}]$ with mean $\bar{R}=10 \mathrm{~cm}$ and standard deviation $\sigma=5 \mathrm{~cm}$. Polynomial chaos expansion $f^{P}(X)$ for different maximum polynomial orders $P$.

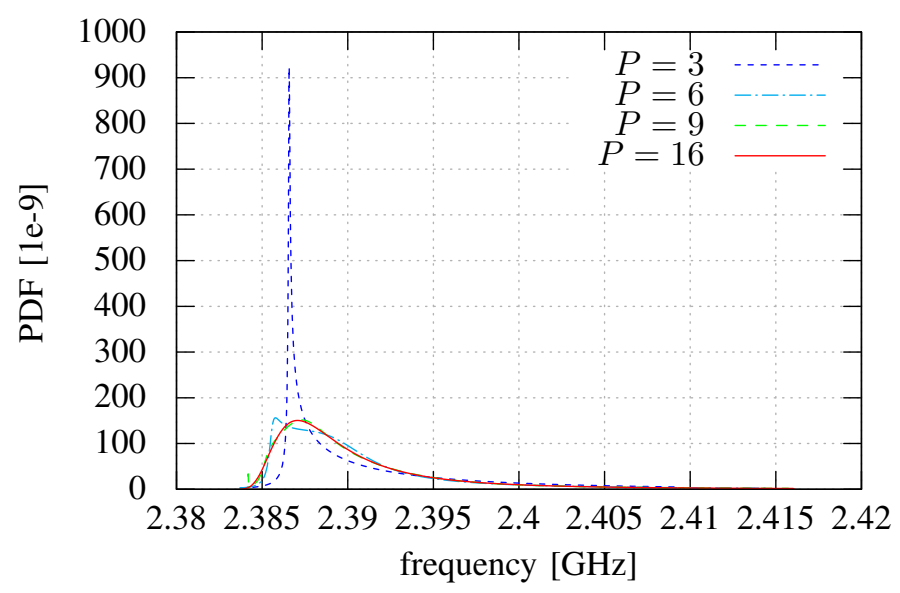

Fig. 8. Prototype 4 bent around a human leg with a radius of curvature following a Gaussian distribution truncated to the interval $[2 \mathrm{~cm}, 30 \mathrm{~cm}]$ with mean $\bar{R}=10 \mathrm{~cm}$ and standard deviation $\sigma=5 \mathrm{~cm}$. PDF of the output random variable for different maximum polynomial orders $P$.

distribution asymmetrical, exhibiting a positive skew of 0.35 . The modified Chebychev algorithm is then applied to generate the appropriate basis of orthogonal polynomials after which the $N=33$ quadrature points follow from the Golub-Welsch algorithm. The output PDF of the resonance frequency shown in Fig. 6, generated using the basis of on-the-fly constructed polynomials to construct the generalized polynomial expansion (1) of order up to $P=16$, is in excellent agreement with the histogram generated using the Monte Carlo data. The PDF now exhibits a skewness of 2.004 (2.026 based on the Monte Carlo simulation).

To investigate the convergence of the generalized polynomial chaos expansion (1) as a function of the maximum polynomial order $P$, we consider prototype 4 , which is a textile patch antenna resonating close to the $2.45 \mathrm{GHz}$ ISM band. We assume that the antenna is bent along the $W_{p}$ direction around a human leg, and that the radius of curvature follows a truncated Gaussian in the interval $[2 \mathrm{~cm}, 30 \mathrm{~cm}]$ with mean $\bar{R}=10 \mathrm{~cm}$ and standard deviation $\sigma=5 \mathrm{~cm}$. In Fig. 7 we plot 
TABLE II

STATISTICAL PROPERTIES OF THE RESONANCE FREQUENCIES WHEN POSITIONING DIFFERENT PROTOTYPES ON A HUMAN ARM WITH A RADIUS OF CURVATURE FOLLOWING A GAUSSIAN DISTRIBUTION TRUNCATED TO THE INTERVAL [2 CM, $9 \mathrm{CM}$ ] WITH MEAN $\bar{R}=5$ CM AND STANDARD DEVIATION $\sigma=2 \mathrm{CM}$. (NUMBERS BETWEEN PARENTHESES: RESULTS OBTAINED BY MONTE CARLO SIMULATIONS)

\begin{tabular}{|c||c||c|c||}
\hline $\begin{array}{c}\text { Proto- } \\
\text { type }\end{array}$ & median & $\begin{array}{c}\text { percentile } \\
5^{\text {th }}\end{array}$ & $\begin{array}{c}\text { percentile } \\
95^{\text {th }}\end{array}$ \\
\hline \hline 1 & $1.5701 \mathrm{GHz}$ & $1.57004 \mathrm{GHz}$ & $1.57037 \mathrm{GHz}$ \\
& $(1.5701 \mathrm{GHz})$ & $(1.57004 \mathrm{GHz})$ & $(1.57036 \mathrm{GHz})$ \\
\hline 2 & $1.58194 \mathrm{GHz}$ & $1.57989 \mathrm{GHz}$ & $1.58707 \mathrm{GHz}$ \\
& $(1.58194 \mathrm{GHz})$ & $(1.57989 \mathrm{GHz})$ & $(1.58703 \mathrm{GHz})$ \\
\hline 3 & $1.55299 \mathrm{GHz}$ & $1.54975 \mathrm{GHz}$ & $1.56098 \mathrm{GHz}$ \\
& $(1.55297 \mathrm{GHz})$ & $(1.54975 \mathrm{GHz})$ & $(1.56092 \mathrm{GHz})$ \\
\hline 4 & $2.3964 \mathrm{GHz}$ & $2.39113 \mathrm{GHz}$ & $2.40942 \mathrm{GHz}$ \\
& $(2.39637 \mathrm{GHz})$ & $(2.39114 \mathrm{GHz})$ & $(2.40948 \mathrm{GHz})$ \\
\hline 5 & $1.53585 \mathrm{GHz}$ & $1.53124 \mathrm{GHz}$ & $1.54661 \mathrm{GHz}$ \\
& $(1.53584 \mathrm{GHz})$ & $(1.53123 \mathrm{GHz})$ & $(1.54673 \mathrm{GHz})$ \\
\hline
\end{tabular}

the function $f^{P}(X)$, transforming the input random variable, being the bending radius $R$, to the output random variable, being the resonance frequency $f_{r}$, for different polynomial orders $P$. In the process, we reduced the number of quadrature points from 33 to 19 , as it was found that this does not significantly reduce the accuracy of the expansion coefficients. These 19 quadrature points used in (4) are also plotted in Fig 7. Fig. 8 shows the corresponding effect of the expansion order $P$ on the probability density function. One notices that the expansion up to order $P=3$ only, provides a poor fit to the output distribution, whereas for $P=6$ and $P=9$, the approximation starts to converge to the actual PDF, exhibiting Gibbs-like oscillations that decrease with higher values of $P$. For $P=16$, the PDF has fully converged to the correct function $Y=f(X)$.

Table II provides the median resonance frequency and the $90 \%$ confidence interval, bounded by the $5^{\text {th }}$ and $95^{\text {th }}$ percentiles when bending the different textile antenna prototypes along the $W_{p}$ direction around a human arm with a radius of curvature following a Gaussian distribution restricted to the interval $[2 \mathrm{~cm}, 9 \mathrm{~cm}]$ with mean $\bar{R}=5 \mathrm{~cm}$ and standard deviation $\sigma=2 \mathrm{~cm}$. The resonance frequency of prototype 1 at $1.57 \mathrm{GHz}$ in planar state exhibits a negligible shift to a median frequency of $1.5701 \mathrm{GHz}$ with a very narrow $90 \%$ confidence interval spanning $320 \mathrm{kHz}$. The fact that the resonance frequency remains very stable when bending the antenna results from the use of the stretchable electro-textile Flectron as conductive material for antenna patch and ground plane. When applying copper foil for the antenna patch and the ground plane, as for antenna prototype 2 , we notice a significant shift in resonance frequency from $1.576 \mathrm{GHz}$ in planar state to a median resonance frequency of $1.58194 \mathrm{GHz}$ in bent state, with a $90 \%$ confidence interval spanning $7.2 \mathrm{MHz}$. Similar behavior is observed for the cotton-based prototype 3 where the resonance frequency of $1.5435 \mathrm{GHz}$ in planar state shifts to a median resonance frequency of $1.55299 \mathrm{GHz}$ in bent state, with a $90 \%$ confidence interval spanning $11.2 \mathrm{MHz}$. Prototype 5, for which a thicker antenna substrate was used,
TABLE III

STATISTICAL PROPERTIES OF THE RESONANCE FREQUENCIES WHEN POSITIONING DIFFERENT PROTOTYPES ON A HUMAN LEG WITH A RADIUS OF CURVATURE FOLLOWING A GAUSSIAN DISTRIBUTION TRUNCATED TO THE INTERVAL [ $2 \mathrm{CM}, 30 \mathrm{CM}$ ] WITH MEAN $\bar{R}=10 \mathrm{CM}$ AND STANDARD DEVIATION $\sigma=5 \mathrm{CM}$. (NUMBERS BETWEEN PARENTHESES: RESULTS OBTAINED By MONTE CARLO SIMULATIONS)

\begin{tabular}{|c||c||c|c||}
\hline $\begin{array}{c}\text { Proto- } \\
\text { type }\end{array}$ & median & $\begin{array}{c}\text { percentile } \\
5^{\text {th }}\end{array}$ & $\begin{array}{c}\text { percentile } \\
95^{\text {th }}\end{array}$ \\
\hline \hline 1 & $1.57002 \mathrm{GHz}$ & $1.57001 \mathrm{GHz}$ & $1.57019 \mathrm{GHz}$ \\
& $(1.57002 \mathrm{GHz})$ & $(1.57001 \mathrm{GHz})$ & $(1.57019 \mathrm{GHz})$ \\
\hline 2 & $1.57903 \mathrm{GHz}$ & $1.57773 \mathrm{GHz}$ & $1.5841 \mathrm{GHz}$ \\
& $(1.57903 \mathrm{GHz})$ & $(1.57773 \mathrm{GHz})$ & $(1.5841 \mathrm{GHz})$ \\
\hline 3 & $1.54838 \mathrm{GHz}$ & $1.54629 \mathrm{GHz}$ & $1.55638 \mathrm{GHz}$ \\
& $(1.54839 \mathrm{GHz})$ & $(1.5463 \mathrm{GHz})$ & $(1.55645 \mathrm{GHz})$ \\
\hline 4 & $2.3889 \mathrm{GHz}$ & $2.38553 \mathrm{GHz}$ & $2.40192 \mathrm{GHz}$ \\
& $(2.38889 \mathrm{GHz})$ & $(2.38554 \mathrm{GHz})$ & $(2.40192 \mathrm{GHz})$ \\
\hline 5 & $1.52925 \mathrm{GHz}$ & $1.52619 \mathrm{GHz}$ & $1.54052 \mathrm{GHz}$ \\
& $(1.52927 \mathrm{GHz})$ & $(1.52617 \mathrm{GHz})$ & $(1.5405 \mathrm{GHz})$ \\
\hline
\end{tabular}

exhibits a larger resonance frequency shift from $1.522 \mathrm{GHz}$ in planar state to a median resonance frequency of $1.53585 \mathrm{GHz}$ in bent state, with a broader $90 \%$ confidence interval spanning 15.4 MHz. Prototype 4, operating close to the $2.45 \mathrm{GHz}$ ISM band with a resonance frequency of $2.381 \mathrm{GHz}$, has its resonance frequency shifted to a median resonance frequency of $2.3964 \mathrm{GHz}$ in bent state, with a $90 \%$ confidence interval spanning $18.3 \mathrm{MHz}$.

Table III presents the median resonance frequency and the 90\% confidence interval, when bending the different textile antenna prototypes along the $W_{p}$ direction around a human leg with a radius of curvature following a Gaussian distribution restricted to the interval $[2 \mathrm{~cm}, 30 \mathrm{~cm}]$ with mean $\bar{R}=10 \mathrm{~cm}$ and standard deviation $\sigma=5 \mathrm{~cm}$. Similar conclusions can be drawn as for the antennas deployed on the arm.

\section{B. Experimental validation}

In the previous subsection, the proposed technique was validated for different probability density functions for the curvature radius of textile antennas. However, in real-life applications, this distribution is not readily available and must be fitted to measured sample data. We now demonstrate how this fitted analytical PDF then forms the starting point for our technique.

Consider the design of a textile antenna, similar to prototype 2, that has to be worn on a human arm. As we focus on the fluctuations in arm radius, the PDF of the arm radius for a representative population needs to be determined. This distribution is derived from measurement campaigns, such as NHANES [27], that provide databases of sample data. Fig. 9 shows the histogram of the arm radius after measuring the arm circumference of 7,056 persons. It is compiled using freely available NHANES examination data of 2009-2010, selecting persons larger than $1.40 \mathrm{~m}$ and heavier than $40 \mathrm{~kg}$. The histogram of the sampled measurement data is now approximated by an analytical PDF, where a truncated normal distribution in the interval $[3 \mathrm{~cm}, 8 \mathrm{~cm}]$, with mean $\bar{R}=5.14 \mathrm{~cm}$ 


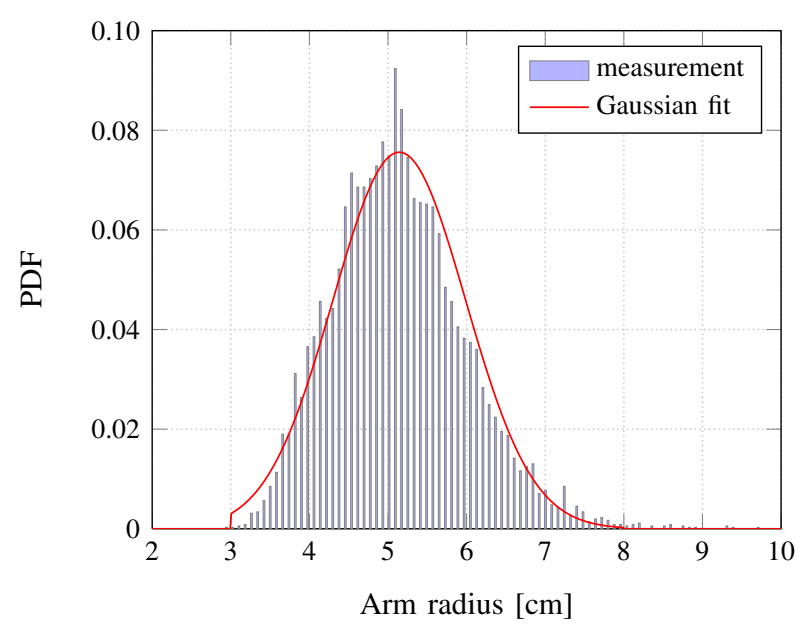

Fig. 9. PDF of the arm radius of different people. NHANES data and truncated Gaussian in $[3 \mathrm{~cm}, 8 \mathrm{~cm}]$ with mean $\bar{R}=5.14 \mathrm{~cm}$ and $\sigma=0.85 \mathrm{~cm}$.

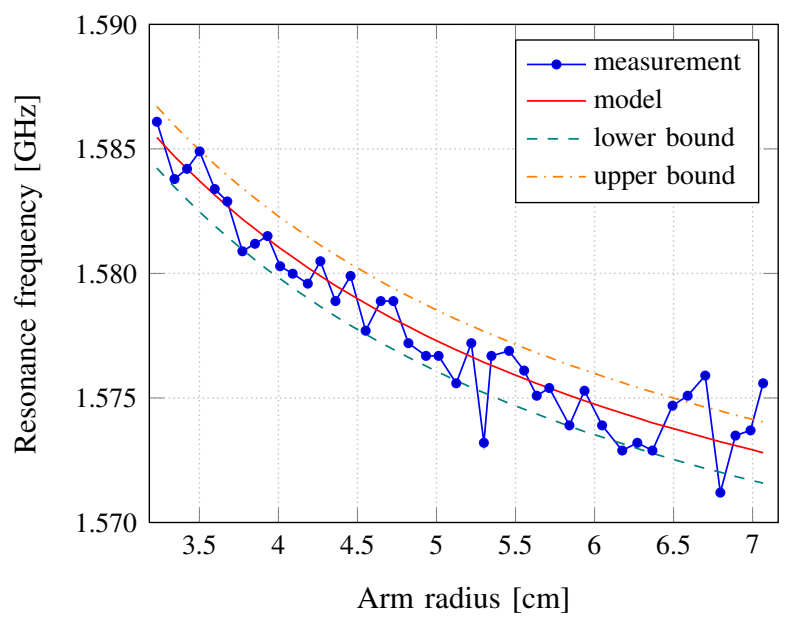

Fig. 10. Resonance frequency as a function of the arm radius. Measurement data and fit.

and $\sigma=0.85 \mathrm{~cm}$ provides a good fit. Remark that these parameters are in good agreement with the parameters used for numerical validation in Section II-A. The fitted truncated Gaussian distribution is also displayed in Fig. 9.

Next, a measurement campaign was performed to construct dispersion relation (6) that relates the arm radius to the measured antenna resonance frequency. Therefore, a new textile antenna prototype was built and the resonance frequencies of the antenna in bent state were measured for different curvature radii, as displayed in Fig. 10. In order to find a good fit, the relative permittivity after compression, $\epsilon_{r, \text { comp }}=$ $\epsilon_{r, \text { flat }}\left(1+\eta \frac{h[m m](d-0.5)}{R[m]}\right)$, was optimized for both $\epsilon_{r, \text { flat }}$ and $\eta$ by applying the least-squares method. The fitted dispersion curve is shown in Fig. 10 by the full line. For error analysis, we also plotted the dispersion relation for $\epsilon_{r, \text { comp }} \pm 0.003$.

The resulting PDF of the resonance frequency is displayed in Fig. 11. The measured data are obtained by solving the fitted dispersion relation (6) with the optimized $\epsilon_{r, \text { comp }}$ for all 7,056 arm radii of the NHANES data set, requiring $59 \mathrm{~s}$ of CPUtime, whereas only 19 evaluations were needed to construct the

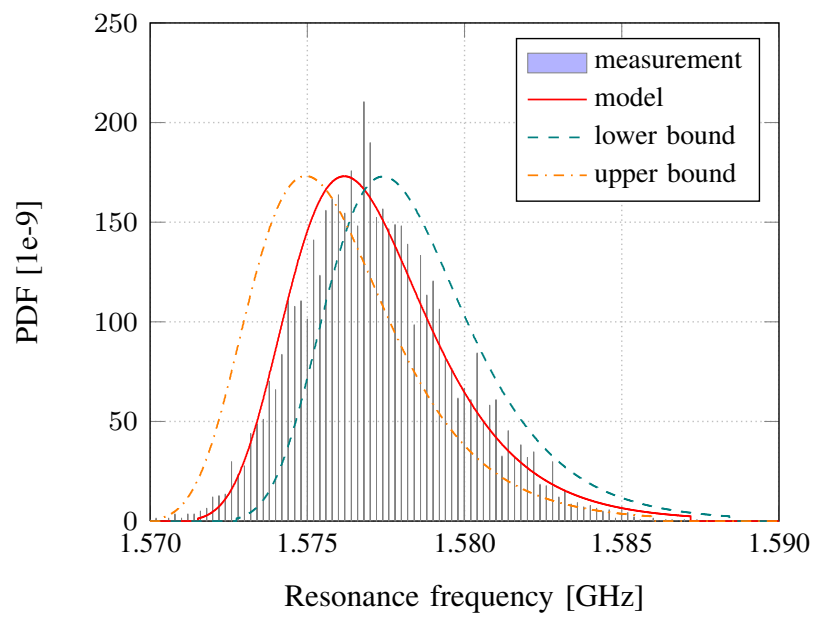

Fig. 11. PDF of the resonance frequency. Measurement and fit.

modelled PDF, taking $0.5 \mathrm{~s}$ of CPU-time (with expansion order $P=9$ ). A good agreement is found between the modelled and the measured data. The modelled PDFs are also shown for $\epsilon_{r, \text { comp }} \pm 0.003$ and one observes a shift of approximately $1 \mathrm{MHz}$ due to the tolerance on the permittivity. Remark that the terms 'lower bound' and 'higher bound' correspond to the bounds defined in Fig. 10.

\section{CONCLUSION}

A new efficient stochastic design tool was introduced based on a non-intrusive generalized polynomial chaos expansion combined with a cavity model for textile antennas subject to cylindrical bending. The method predicts the cumulative distribution function, the probability density function, the confidence intervals and the moments of the statistical distribution of the resonance frequency when the radius of curvature of the textile patch is a random variable. The method can be applied to input distributions of the Askey family as well as to any arbitrary distribution. For the latter, the Modified Chebyshev algorithm is implemented to construct the relevant set of orthogonal polynomials, followed by the Golub-Welsch algorithm to determine the quadrature points. The dispersion relation of the cavity model is then solved for realizations corresponding to these points. Comparison to Monte Carlo simulations and to experimental data demonstrates the accuracy and the large speedup of at least a factor 100 obtained by the new procedure.

\section{ACKNOWLEDGMENT}

The work of F. Boeykens was supported by a doctoral grant from the Agency for Innovation by Science and Technology in Flanders (IWT). Part of this work was supported by BELSPO through the IAP Phase VII BESTCOM project. The authors would also like to thank prof. D. De Zutter for proofreading the manuscript.

\section{REFERENCES}

[1] A. Tronquo, H. Rogier, C. Hertleer, and L. Van Langenhove, "Robust planar textile antenna for wireless body LANs operating in $2.45 \mathrm{GHz}$ ISM band," IEE Electronic Letters, vol. 42, no. 3, pp. 142-146, 2006. 
[2] I. Locher, M. Klemm, T. Kirstein, and G. Troster, "Design and characterization of purely textile patch antennas," IEEE Transactions on Advanced Packaging, vol. 29, no. 4, pp. 777-788, December 2006.

[3] P. Salonen and Y. Rahmat-Samii, "Textile antennas: Effects of antenna bending on input matching and impedance bandwidth," IEEE Aerospace and Electronic Systems Magazine, vol. 22, no. 12, pp. 18-22, December 2007.

[4] C. Hertleer, H. Rogier, L. Vallozzi, and L. Van Langenhove, "A textile antenna for off-body communication integrated into protective clothing for firefighters," IEEE Trans. on Antennas and Propagation, vol. 57, no. 4, pp. 919-925, April 2009.

[5] Q. Bai and R. Langley, "Crumpling of PIFA textile antenna," IEEE Trans. on Antennas and Propagation, vol. 60, no. 1, pp. 63-70, January 2012.

[6] D. Xiu and G. E. Karniadakis, "The wiener-askey polynomial chaos for stochastic differential equations," SIAM J. Sci. Comput, vol. 24, no. 2, pp. 619-644, 2002.

[7] J. Shen, H. Yang, and J. Chen, "Analysis of Electrical Property Variations for Composite Medium Using a Stochastic Collocation Method," IEEE TRANSACTIONS ON ELECTROMAGNETIC COMPATIBILITY, vol. 54, no. 2, pp. 272-279, APR 2012.

[8] O. O. Sy, M. C. Van Beurden, B. L. Michielsen, J. Vaessen, and A. Tijhuis, "Second-order statistics of complex observables in fully stochastic interactions: applications to EMC," Radio Science, vol. 45, no. RS4004, pp. 1-16, 2010.

[9] M. S. Gilbert and F. L. Teixeira, "A Small-Perturbation AutomaticDifferentiation Method for Determining Uncertainty in Computational Electromagnetics," IEEE TRANSACTIONS ON ANTENNAS AND PROPAGATION, vol. 60, no. 11, pp. 5305-5314, NOV 2012.

[10] D. Xiu, "Fast numerical methods for stochastic computations: A review," Commun. Comput. Phys, vol. 5, no. 2-4, pp. 242-272, February 2009.

[11] D. Vande Ginste, D. De Zutter, D. Deschrijver, T. Dhaene, P. Manfredi, and F. Canavero, "Stochastic modeling-based variability analysis of on-chip interconnects," IEEE Trans. On Components, Packaging And Manufacturing Technology, vol. 2, no. 7, pp. 1182-1192, July 2012.

[12] P. Manfredi and F. G. Canavero, "Polynomial chaos for random field coupling to transmission lines," IEEE Trans. On Electromagnetic Compatibility, vol. 54, no. 3, pp. 677-680, June 2012.

[13] H. Bagci, A. Yucel, J. Hesthaven, and E. Michielssen, "A fast Stroudbased collocation method for statistically characterizing EMI/EMC phenomena on complex platforms," IEEE Trans. Electromagn. Compat., vol. 51, no. 2, pp. 301-311, May 2009.

[14] A. Biondi, D. V. Ginste, D. De Zutter, P. Manfredi, and F. G. Canavero, "Variability Analysis of Interconnects Terminated by General Nonlinear Loads," IEEE TRANSACTIONS ON COMPONENTS PACKAGING AND MANUFACTURING TECHNOLOGY, vol. 3, no. 7, pp. 1244-1251, JUL 2013.

[15] P. Sumant, H. Wu, A. Cangellaris, and N. Aluru, "Reduced-order models of finite element approximations of electromagnetic devices exhibiting statistical variability," IEEE Trans. on Antennas and Propagation, vol. 60, no. 1, pp. 301-309, January 2012.

[16] D. Spina, F. Ferranti, T. Dhaene, L. Knockaert, G. Antonini, and D. Vande Ginste, "Variability analysis of multiport systems via polynomial-chaos expansion," IEEE Trans. on Microwave Theory and Techniques, vol. 10, no. 1109, TMTT.2012.2202685, 2012.

[17] A. Austin and C. Sarris, "Efficient analysis of geometrical uncertainty in the FDTD method using polynomial chaos with application to microwave circuits," Microwave Theory and Techniques, IEEE Transactions on, vol. Early Acess Paper, pp. 1-9, 2013.

[18] C. Chauviere, J. Hesthaven, and L. Lurati, "Computational modeling of uncertainty in time-domain electromagnetics," in Computational Electromagnetics in Time-Domain, 2005. CEM-TD 2005. Workshop on, 2005, pp. 32-35.

[19] J. S. Ochoa and A. C. Cangellaris, "Macro-Modeling of Electromagnetic Domains Exhibiting Geometric and Material Uncertainty," $A P$ PLIED COMPUTATIONAL ELECTROMAGNETICS SOCIETY JOUR$N A L$, vol. 27, no. 2, SI, pp. 80-87, FEB 2012.

[20] J. Ochoa and A. Cangellaris, "Expedient electromagnetic analysis of the impact of statistical disorder in periodic waveguides," Microwave Theory and Techniques, IEEE Transactions on, vol. 60, no. 12, pp. 3919-3926, 2012.

[21] F. Boeykens, L. Vallozzi, and H. Rogier, "Cylindrical bending of deformable textile rectangular patch antennas," International Journal of Antennas and Propagation, vol. 420, no. 11, pp. 1-11, Article ID 170, doi:10.1155/2012/170 420, 2012.
[22] G. Poëtte and D. Lucor, "Non intrusive iterative stochastic spectral representation with application to compressible gas dynamics," Journal of Computational Physics, vol. 231, no. 9, pp. 3587-3609, May 2012.

[23] X. Wan and G. E. Karniadakis, "Beyond Wiener-Askey expansions: Handling arbitrary PDFs,” J. Sci. Comput, vol. 27, no. 1-3, pp. 455-464, 2006.

[24] W. Gautschi, Orthogonal Polynomials - Computation and Approximation. Oxford: ISBN 0198506724, p.76, 2004.

[25] G. Golub and J. Welsch, "Calculation of Gauss quadrature rules," Mathematics of Computation, vol. 23, no. 106, pp. 221-230, Apr 1969.

[26] Determination of thickness of textiles and textile products, ISO Standard 5084, 1996.

[27] Centers for Disease Control and Prevention. (2011, Sep.) National Health and Nutrition Examination Survey. [Online]. Available: http://www.cdc.gov/nchs/nhanes.htm

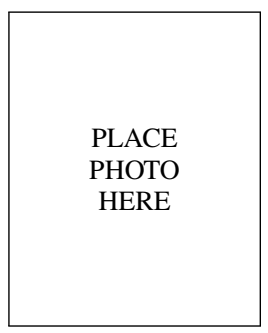

Freek Boeykens was born in 1986 . He received the M.S. and Ph.D. degrees in Electrical Engineering from Ghent University, Gent, Belgium in 2009 and in 2013 respectively. He is currently a researcher at the Department of Information Technology at Ghent University. His research interests comprise numerical electromagnetics and antenna systems.

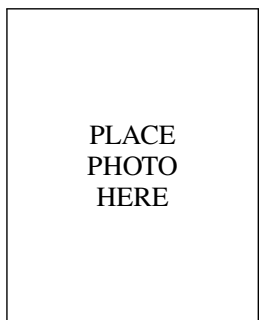

Hendrik Rogier was born in 1971 . He received the Electrical Engineering and the Ph.D. degrees from Ghent University, Gent, Belgium, in 1994 and in 1999, respectively. He is a currently a Full Professor with the Department of Information Technology of Ghent University, Belgium, Guest Professor at IMEC, Heverlee, Belgium, and Visiting Professor at the University of Buckingham, UK. From October 2003 to April 2004, he was a Visiting Scientist at the Mobile Communications Group of Vienna University of Technology. He authored and coauthored about 90 papers in international journals and about 105 contributions in conference proceedings. He is serving as a member of the Editorial Board of IET Science, Measurement Technology and acts as the URSI Commission $B$ representative for Belgium. Within the IEEE Microwave Theory and Techniques Society, he is a member of Technical Committee 24 on RFID technology and within the European Microwave Association, he is a member of the Governing Board of Topical Group MAGEO on Microwaves in Agriculture, Environment and Earth Observation. His current research interests are antenna systems, radiowave propagation, body-centric communication, numerical electromagnetics, electromagnetic compatibility and power/signal integrity.

Dr. Rogier was twice awarded the URSI Young Scientist Award, at the 2001 URSI Symposium on Electromagnetic Theory and at the 2002 URSI General Assembly. He is a Senior Member of the IEEE.



Luigi Vallozzi was born in Ortona, Italy, in 1980. He received the Laurea degree in electronic engineering from the Universit Politecnica delle Marche, Ancona, Italy, in 2005 and the Ph.D. degree in electrical engineering at Ghent University, Ghent, Belgium, in 2010. He is currently working as a Postdoctoral Research Fellow of the Fund for Scientific Research Flanders (FWO) within the Electromagnetics Group at the Information Technology Department (INTEC) of Ghent University. His research focuses on design and prototyping of antennas for wearable textile systems, and the modeling and characterization of multiple-input multipleoutput wireless communication systems. 